\title{
L'énergie marémotrice en 1989
}

\section{Tidal energy in 1989}

par

Michel Banal

Dans le numéro 5/6-1982 de la Houille Blanche, consacré aux aménagements hydroélectriques de grande puissance, nous avions donné, sous le titre "L'énergie marémotrice en 1982 », un panorama des projets et des réalisations de cette énergie dans le monde.

Quelle est la situation en 1989 et qu'est-il advenu des grands projets que nous avions évoqués?

Le bilan est vite fait puisqu'à part l'usine expérimentale d'Annapolis, mise en service en 1984, aucun grand projet n'a reçu un début d'exécution.

Cela ne veut pas dire que toutes les études aient été abandonnées et il a été possible de faire le point de leur avancement à l'occasion notamment de deux réunions internationales tenues respectivement : en mai 1986 à Brighton (UK), et en février 1988 à Delhi (Inde).

Il faut citer aussi la session du comité technique de la Société hydrotechnique de France (SHF) du 15 mars 1984, publiée dans le numéro 8-1984 de La Houille Blanche, où la situation de l'énergie marémotrice en France a été étudiée, ainsi que la réunion tenue à Dinard en novembre 1986 à l'occasion des vingt ans d'exploitation de l'usine de la Rance. Dans ce qui suit ces différentes sources d'information seront évidemment largement utilisées.

\section{Les grands projets}

Ne seront évoqués ici que les projets de plusieurs centaines de milliers de $\mathrm{kW}$ au moins ayant fait l'objet d'études importantes pendant les dix dernières années, c'est-à-dire ceux concernant la baie de Fundy au Canada, le golfe de Kachchh en Inde, la baie de Garolim en Corée du Sud, l'estuaire de la Severn en Grande-Bretagne et la baie de
Saint-Malo en France. Un inventaire plus complet des sites où l'utilisation de l'énergie de la marée a été envisagée ou réalisée était donné dans le tableau $\mathrm{n}^{\circ} 1$ de l'article du numéro de 1982 de La Houille Blanche déjà cité. Nous reproduisons ce tableau à la page suivante.

LA HOUILLE BLANCHE/ $\mathrm{N}^{\circ}$ 6-1989 


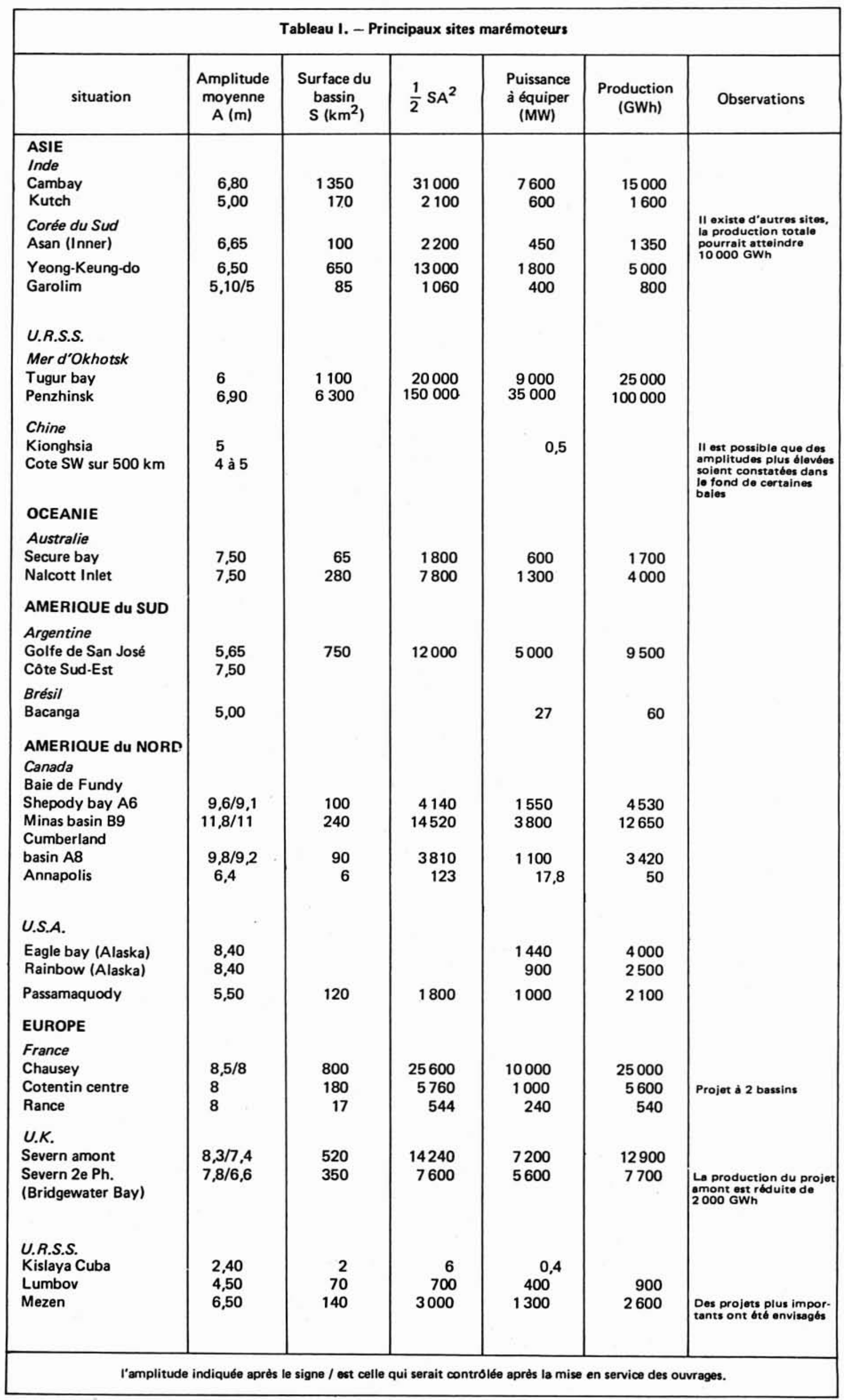


en périphérie de la roue), et l'utilisation ou non du pompage pour remplir le bassin.

Ces deux questions sont partiellement liées, car seuls les groupes à pales orientables peuvent être utilisés en pompage. L'adaptation de l'usine pour le pompage n'entraîne alors qu'une dépense supplémentaire très modeste et ne provoque pas de perte sur le rendement en turbinage.

Au contraire, le fonctionnement en double effet (production au remplissage et à la vidange) exige des dispositions onéreuses ou entraînant des pertes de rendement notables, et pour cette raison ce type de fonctionnement n'est recommandé dans aucun projet.

Il est à noter à ce sujet que si le pompage en fin de remplissage du bassin est régulièrement utilisé à l'usine de la Rance, il n'en est pas de même pour la production au remplissage (turbinage inverse).

\subsection{Baie de Saint-Malo}

A contre courant des projets cités précédemment, la France a étudié au début des années 80 des projets très différents et originaux à plusieurs bassins conjugués permettant une plus grande flexibilité de la production.

D'autre part, la difficulté d'implanter des ouvrages à la côte en raison de son occupation par de multiples activités (pêche, conchyliculture, loisirs, etc...) a conduit à un projet conçu comme un atoll isolé de la côte.

Ceci n'a certes pas contribué à rendre le projet crédible aux décideurs, mais il faut observer en outre que la flexibilité de la production apportée par les cycles complexes à plusieurs bassins ne peut être bien valorisée que s'il est possible de prévoir quels seront dans les vingt ans à venir au moins, suivant les heures du jour et les périodes de l'année, les coûts des autres moyens de production, et bien entendu si ces coûts sont assez différenciés. L'expérience a largement montré qu'une telle prévision était impossible.

Autrement dit, on ne peut pas tirer complètement profit de l'absence d'aléas dans la prévision des marées, en raison de l'incertitude dans la prévision à long terme des conditions d'exploitation du réseau.

Ces études ont été complètement arrêtées en 1983.

\section{L'usine marémotrice d'Annapolis (Canada)}

A vrai dire, il ne s'agit pas vraiment d'une usine marémotrice, mais plutôt d'une station expérimentale comme l'a été le groupe monté dans l'écluse de Saint-Malo en 1959 et exploité jusqu'en 1964, même si la production de ce groupe était négligeable, ce qui n'est pas le cas pour Annapolis.

Son objectif est d'expérimenter en site maritime un groupe de grande puissance à alternateur en périphérie de la roue. Cette technique, issue d'un brevet pris par Haza en 1919 et développé par Escher Wyss sous le nom de Straflo, n'avait jamais été appliquée jusqu'ici qu'en site fluvial, avec des puissances ne dépassant pas $2 \mathrm{MW}$ et un diamètre de roue de $3 \mathrm{~m}$ à $3,70 \mathrm{~m}$.

A Annapolis, la puissance est de 17,8 MW sous $5,50 \mathrm{~m}$ de chute et le diamètre de la roue est de $7,60 \mathrm{~m}$. Il s'agit donc d'une extrapolation très importante par rapport à tout ce qui avait été fait jusqu'ici.

L'intérêt des groupes à alternateurs périphériques est d'éviter les restrictions sur le diamètre de l'alternateur imposées aux groupes bulbes, et de permettre sans doute une légère réduction de la longueur du conduit hydraulique.

Il s'agit en tous cas d'une expérience extrêmement intéressante, qui paraît donner jusqu'ici satisfaction aux exploitants comme au constructeur.

Marnage moyen ............................ $6,5 \mathrm{~m}$
Puissance équipée ........................ $18 \mathrm{MW}$
Production annuelle $\ldots \ldots \ldots \ldots \ldots \ldots \ldots \ldots . . . .65$ millions de $\mathrm{kWh}$

La dimension du bassin aurait justifié une puissance équipée double, mais cela n'aurait pas accru sensiblement l'intérêt de l'expérience.

La faiblesse de l'équipement, les caractéristiques de la turbine, et les restrictions aux variations du niveau du bassin imposées par la protection des terres agricoles rendent l'exploitation de la station d'Annapolis extrêmement simple (par rapport à celle de l'usine de la Rance). 


\section{L'usine de la Rance}

\subsection{Fonctionnement et environnement}

A chacune des occasions déjà rappelées, notamment à la séance du 15 mars 1984 du comité technique de la SHF et à la réunion tenue en 1986 a l'occasion du $20^{\circ}$ anniversaire de sa mise en service, l'excellent fonctionnement de l'usine de la Rance a été abondamment rappelé, ainsi que l'excellent état de ses installations, comme tous les visiteurs, spécialistes ou non, peuvent le constater chaque jour.

Tout ce qu'on peut ajouter à ces nombreux témoignages, c'est que la situation en 1989 est aussi bonne qu'elle l'était pendant les années précédentes, depuis l'achèvement de la remise en état des alternateurs en 1983. Les indisponibilités fortuites des groupes sont extrêmement rares, et, les indisponibilités pour entretien étant concentrées sur les périodes de mortes-eaux oủ la production est plus faible, la perte de production pour indisponibilité du matériel ne dépasse pas un pour cent de la production théorique.

II ne faut pas cacher cependant que de tels résultats n'ont été obtenus que grâce à la vigilance des exploitants. confrontés à des problèmes entièrement nouveaux et dans des domaines extrêmement variés. Il a fallu, par exemple, s'y reprendre de nombreuses fois avant de trouver une solution satisfaisante au montage du filet placé à $200 \mathrm{~m}$ en amont de l'usine pour empêcher les navigateurs imprudents de trop s'en approcher.

L'impact sur l'environnement de l'aménagement de la Rance n’avait pas été particulièrement étudié avant sa construction ; il fait par contre maintenant l'objet d'un suivi attentif, comme en témoignent notamment les articles de C. Rètière, Directeur du Laboratoire maritime de Dinard, dans La Houille Blanche ( $\mathrm{N}^{\circ} 8-1984$ et $\left.\mathrm{N}^{\circ} 2-1989\right)$, qui concluent à un bilan globalement positif.

Le barrage lui-même, la liaison entre les rives de la Rance, la protection du bassin amont qu'il assure sont tellement entrés dans les habitudes et le paysage qu'on imagine mal les protestations que soulèverait - hypothèse invraisemblable - un projet de les faire disparaître.

\subsection{Automatisation de l'exploitation}

Un fait nouveau et important est intervenu pourtant en 1988 à l'usine de la Rance : l'automatisation complète de son exploitation.

Il existe certes déjà en France et dans le monde de très nombreuses usines hydro-électriques dont le fonctionne- ment est largement automatisé, mais pour une usine marémotrice et spécialement une usine ayant les possibilités de celle de la Rance, e'est bien autre chose, en raison de la complexité des cycles de fonctionnement et de la nécessité de les adapter à tout moment aux aléas du réseau et de la marée, car suivant la formule connue : la marée n'attend pas et tout retard dans l'exécution d'un ordre entraîne une perte définitive de production.

Pour expliquer - en simplifiant à l'extrême - l'innovation apportée en 1988 à l'exploitation de l'usine de la Rance, rappelons que la gestion de sa production a comporté dès sa mise en service trois niveaux :

1) L'établissement dans un centre de calcul, grâce à un modèle de gestion très élaboré, du programme hebdomadaire d'exploitation optimisant la production en fonction des cycles de la marée, de la valeur de l'énergie aux différents moments de la période considérée, des contraintes diverses sur les niveaux du bassin et les débits, etc...

2) La lecture de ce programme par un ordinateur installé a l'usine, sa confrontation avec les données locales instantanées (disponibilité des groupes, niveau du bassin, etc...), et l'élaboration des ordres aux groupes et aux vannes.

3) Le relayage primaire assurant suivant ces ordres le démarrage, l'arrêt et le réglage des groupes (par ensemble de 4) ainsi que louverture et la fermeture des vannes.

Le modèle de gestion (premier niveau) a été réécrit en 1980 (programme A.G.R.A.) en utilisant les progrès de l'informatique. Il est exploité depuis lors sur l'IBM 3091 de la Direction des études et recherches d'EDF (6 min de calcul pour une semaine d'exploitation).

C'est le niveau intermédiaire qui vient cette fois-ci d'être refait et ses possibilités sont considérablement accrues.

La vétusté du matériel monté en 1968 et l'impossibilité de se procurer des pièces de rechange imposaient le changement et on en a profité pour en accrôtre les performances de manière à :

- assurer la conduite automatique de l'aménagement sans personnel permanent de conduite ;

- améliorer la qualité de la conduite grâce notamment à une prise en compte plus rapide et plus précise des écarts entre les niveaux constatés et prévus :

- automatiser et étendre la collecte et la diffusion de toutes les informations nécessaires aux exploitants et aux usagers. 


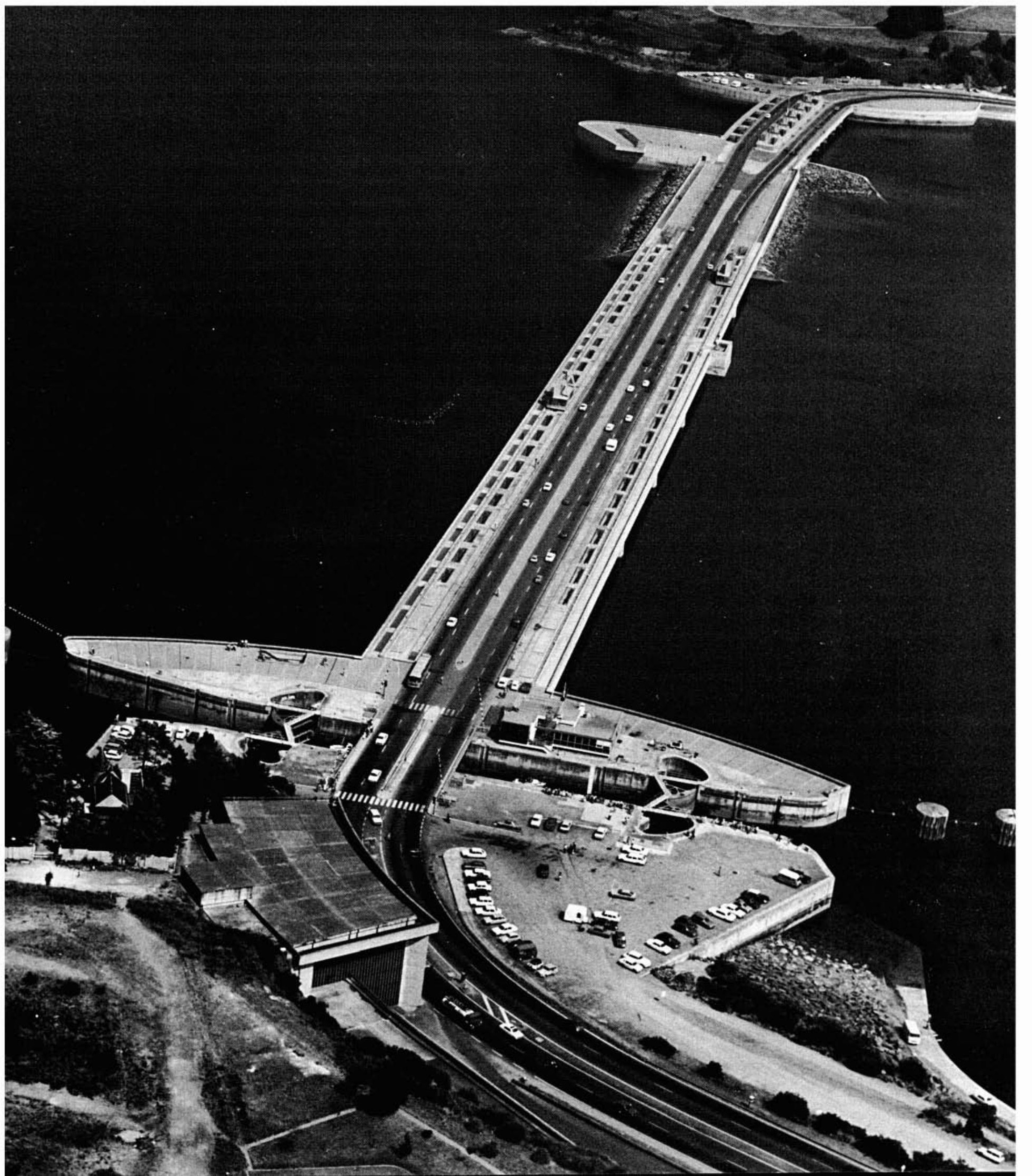


Bien que le matériel ancien ait dû permettre en théorie la transmission aux machines, sans intervention humaine, du programme d'exploitation, les opérations devaient être en permanence contrôlées par le personnel de quart, vérifiant que les ordres de l'ordinateur étaient correctement exécutés, assurant directement les corrections au programme nécessitées notamment par les écarts entre les niveaux prévus et constatés du bassin, relevant les anomalies de fonctionnement et toutes les données nécessaires au contrôle a posteriori de l'exploitation.

Pour obtenir un véritable automatisme de fonctionnement de l'usine. les dispositions suivantes ont été adoptées pour les nouvelles installations :

1) Doublement de l'ordinateur, avec reprise automatique en secours d'un ordinateur par l'autre.

2) Création entre les ordinateurs et le relayage de commande des groupes et des vannes d'un niveau intermédiaire d'automatismes programmables (un automate pour 4 groupes et un automate pour l'ensemble des vannes) en mesure d'assurer le suivi du programme d'exploitation pendant deux heures en cas d'indisponibilité des ordinateurs ou des liaisons avec eux.

3) Multiplication des installations périphériques assurant en continu la collecte, le traitement, la diffusion et le stockage de toutes les informations sur le fonctionnement de l'usine, y compris celles concernant la protection cathodique dont le rôle dans la bonne conservation des structures métalliques est essentiel.
L'ensemble le plus important de ces terminaux se trouve évidemment dans la salle de commande de l'usine, mais les informations sont également transmises dans le bâtiment d'exploitation et aussi au bureau du port de Saint-Malo et à l'écluse du Chatelier, limite amont du bassin.

Parmi les fonctions essentielles que le nouveau système de conduite est en mesure d'assurer, il faut citer :

- le "microrattrapage" c'est-à-dire la modification de quelques degrés de l'incidence des pales prescrite par l'ordinateur pour résorber l'écart constaté entre les niveaux réel et prévu du bassin ;

- l'"assistance au démarrage" par un accroissement temporaire de l'incidence des pales des groupes dont la vitesse de rotation n'est pas suffisante (ou dans le bon sens) au moment de l'envoi d'un ordre de démarrage.

Mais il faudrait citer aussi les multiples contrôles et vérifications effectués sans intervention humaine à tous les stades de l'exécution du programme d'exploitation.

Les installations dont les performances ont été briẻvement décrites ont été commandées en septembre 1984 par la Division électricité du Service de la production hydraulique d'EDF, maitre-d'œuvre, à la Société Sema-Metra associè à SPIE-Batignolles. Leur mise en service sur le site a été effectuée en octobre 1987, et depuis l'été 1988 la centrale est exploitee conformément aux objectifs que s'étaient fixés les responsables du projet.

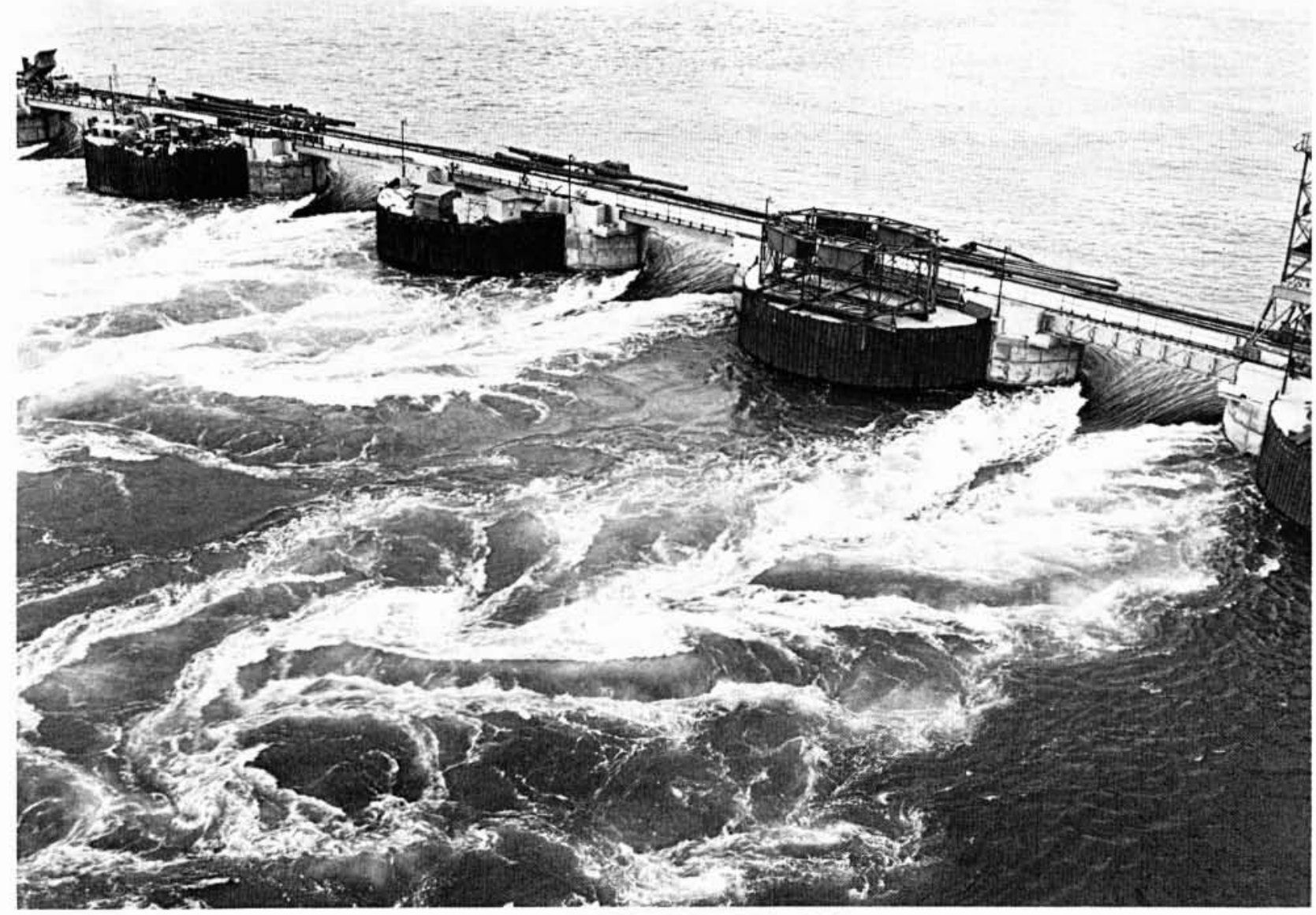




\section{Avenir de l'énergie marémotrice}

Ayant été invité à faire l'exposé introductif d'une des séances du Congrès de Delhi, je l'avais titré d'une manière intentionnellement provocante: Qu'est-ce qui est le plus important pour le succès d'un projet d'usine marémotrice, l'amplitude de la marée ou l'environnement?

Il est bien entendu hors de question de sous-estimer l'influence de l'amplitude de la marée sur l'économie d'une usine marémotrice, mais ceci est tellement connu que cette amplitude est souvent considérée comme le seul critère de choix d'un site alors qu'il apparait de plus en plus que les conditions d'environnement sont aussi et peut-être plus importantes si l'on y inclut à la fois le coût des autres sources d'énergie avec lesquelles l'usine projetée est en compétition et les inconvénients et avantages que les ouvrages peuvent apporter à la collectivité en dehors de la production d'électricité.

Il est certain par exemple que le projet de l'usine de la Rance aurait été beaucoup moins facilement accepté s'il n'avait pas assuré à bon compte une liaison rapide entre Dinard et Saint-Malo.

Ce qui était vrai dans les années 50 l'est certainement encore bien plus actuellement, et un projet d'usine marémotrice n'aura, à mon avis, des chances d'aboutir que s'il s'intègre dans l'environnement terrestre et maritime d'une manière globalement positive pour les populations environnantes.
Encore faut-il que les avantages du projet (liaisons routières, bassins protégés, îles artificielles, etc...) soient mis en évidence. Or s'il se trouve toujours beaucoup de gens pour déceler et prédire les inconvénients et les risques d'un projet, seul son promoteur peut découvrir et valoriser les avantages que les mêmes ouvrages peuvent présenter pour les populations avoisinantes, et en faire un véritable projet à buts multiples. Si au contraire il se borne à une attitude défensive à l'égard des opposants, les chances d'aboutir resteront très modestes.

La première conséquence qu'on doit tirer de ce qui précède c'est que la recherche des sites aptes à l'utilisation des marées doit s'étendre bien au-delà des sites connus pour leurs marées exceptionnelles et il est impossible de fixer une limite inférieure du marnage au-dessous de laquelle un projet d'usine marémotrice perd toute chance. Tout dépend de son environnement.

Une deuxième conséquence est l'importance pour le succès des projets marémoteurs des progrès qui pourront être faits sur l'utilisation des très basses chutes. Les groupes à alternateurs en périphérie de la roue ont été cités précédemment, mais il existe d'autres développements et notamment celui des groupes bulbes à multiplicateur.

L'environnement, enfin, au sens large défini précédemment, doit être, dès l'origine, la principale préoccupation du promoteur du projet. 